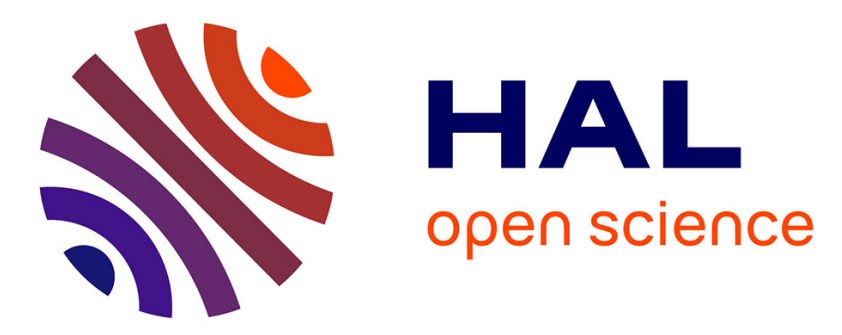

\title{
Turbulent channel without boundaries: The periodic Kolmogorov flow
}

S Musacchio, G Boffetta

\section{To cite this version:}

S Musacchio, G Boffetta. Turbulent channel without boundaries: The periodic Kolmogorov flow. Physical Review E: Statistical, Nonlinear, and Soft Matter Physics, 2014, 89, pp.23004. 10.1103/PhysRevE.89.023004 . hal-01082279

\section{HAL Id: hal-01082279 \\ https://hal.univ-cotedazur.fr/hal-01082279}

Submitted on 13 Nov 2014

HAL is a multi-disciplinary open access archive for the deposit and dissemination of scientific research documents, whether they are published or not. The documents may come from teaching and research institutions in France or abroad, or from public or private research centers.
L'archive ouverte pluridisciplinaire HAL, est destinée au dépôt et à la diffusion de documents scientifiques de niveau recherche, publiés ou non, émanant des établissements d'enseignement et de recherche français ou étrangers, des laboratoires publics ou privés. 


\title{
Turbulent channel without boundaries: The periodic Kolmogorov flow
}

\author{
S. Musacchio \\ Laboratoire J. A. Dieudonné, UMR No. 7351, Université de Nice Sophia Antipolis, CNRS, 06100 Nice, France \\ G. Boffetta \\ Dipartimento di Fisica and INFN, Università di Torino, Via P. Giuria 1, 10125 Torino, Italy
}

(Received 13 December 2013; published 7 February 2014)

\begin{abstract}
The Kolmogorov flow provides an ideal instance of a virtual channel flow: It has no boundaries, but it possesses well defined mean flow in each half wavelength. We exploit this remarkable feature for the purpose of investigating the interplay between the mean flow and the turbulent drag of the bulk flow. By means of a set of direct numerical simulations at increasing Reynolds number, we show the dependence of the bulk turbulent drag on the amplitude of the mean flow. Further, we present a detailed analysis of the scale-by-scale energy balance, which describes how kinetic energy is redistributed among different regions of the flow while being transported toward small dissipative scales. Our results allow us to obtain an accurate prediction for the spatial energy transport at large scales.
\end{abstract}

DOI: 10.1103/PhysRevE.89.023004

PACS number(s): 47.27.E-, 47.27.W-

\section{INTRODUCTION}

Theoretical and numerical studies of turbulent flows can be divided into two categories. The first class of studies, motivated mainly by experiments and practical applications, considers turbulence as generated by the interaction of the flow with a solid object. The simplest, and most widely studied, example is the interaction with a plane, as in the turbulent channel. The other category focuses mainly on intrinsic properties of turbulence: bulk quantities, which may be expected to give universal statistics independently of the way the flow is generated. These studies are usually based on the simplest possible geometry in the absence of boundaries, the so-called homogeneous-isotropic turbulence in periodic domains.

Between these two widely studied classes, there is another class of inhomogeneous flow in the absence of boundaries. In these flows, of which the Kolmogorov flow is the most studied example, homogeneity and isotropy are broken not by physical boundaries but by the body force that generates the flow. This flow was proposed by Kolmogorov as a model to understand the transition to turbulence and was first studied by his students who showed that the laminar solution becomes unstable to large-scale perturbations at the critical Reynolds number $\operatorname{Re}=\sqrt{2}$. Further studies investigated analytically the evolution of the perturbation just above the instability [1] and numerically the transition to turbulence [2-4]. Because it is very convenient for analytical studies and numerical simulations, the Kolmogorov flow has also been used for several investigations in anisotropic and/or inhomogeneous conditions, e.g., to investigate the anisotropy decompositions of turbulent flows [5,6], the instabilities in the presence of Rossby waves [7], stratification [8], and viscoelastic solution $[9,10]$. Another important example is the Taylor-Green vortex, which is closely related to the von Kármán flow used in experimental studies of hydrodynamic and magnetohydrodynamic turbulence. The different symmetries of these flows makes them suitable to investigate different classes of questions. The Taylor-Green flow is characterized by a shear region between two counterrotating vortices and has been widely used in numerical studies of magnetohydrodynamic dynamos (see, e.g., [11,12]).
The Kolmogorov flow can be thought of as a simplified channel flow without boundaries. It displays a mean velocity profile that vanishes at the nodes of the sinusoidal force. Therefore, it can be seen as a series of virtual channels, whose width is equal to a half period of the forcing, flowing in alternate directions without being confined by material boundaries. In contrast, because of the lack of boundaries, in the Kolmogorov flow the complex flow structures produced by the wall, injected into the bulk, and responsible for the energy transfer in bounded channel flows are missing. For these reasons the periodic Kolmogorov flow allows one to isolate bulk properties, e.g., of the turbulent drag, which in a real channel flow might be hidden by the complex near-wall phenomenology. In this spirit it has been used recently to study the drag-reduction phenomenon induced by polymer additives [13].

The drag coefficient, or friction factor, is defined as the ratio between the work made by the force and the kinetic energy carried by the mean flow. This fundamental dimensionless number measures the power that has to be supplied to the fluid to maintain a given throughput. In general, when the flow is laminar, the drag coefficient is inversely proportional to the Reynolds number. Upon increasing the intensity of the applied force, the flow eventually becomes turbulent and the drag coefficient becomes approximately independent of the Reynolds number Re and therefore substantially larger than in the laminar case.

No exact values for the friction factor are known, even in a simple geometry. In the case of smooth pipe flows, an empirical logarithmic formula (the Colebrook-White equation) reproduces accurately the experimental data. From a different perspective, rigorous mathematical bounds have been derived for the friction factor with different geometries, also for the Kolmogorov flow [14]. In spite of their importance from a theoretical point of view, they are not strongly constrictive and therefore not very useful for applications.

Here we present the results of numerical simulations of the turbulent Kolmogorov flow aimed to study the dependence of turbulent drag on the Reynolds number. We also present a detailed analysis of the scale-by-scale energy balance that shows how the kinetic energy is redistributed among different 
regions and different scales of each virtual channel. Moreover, we discuss the statistics of small-scale velocity fluctuations and the scaling of structure functions in the inertial range of scales.

\section{PHENOMENOLOGY OF THE KOLMOGOROV FLOW}

We consider the Navier-Stokes equations for an incompressible velocity field $u_{i}(\mathbf{x}, t)(i=1,2,3)$,

$$
\partial_{t} u_{i}+u_{j} \partial_{j} u_{i}=-\partial_{i} p+v \partial^{2} u_{i}+g_{i},
$$

forced by the Kolmogorov body force $g_{i}=\delta_{i, 1} F \cos (z / L)$. Equation (1) admits a stationary solution, the laminar Kolmogorov flow $u_{i}=\delta_{i, 1} U_{0} \cos (z / L)$ with $F=v U_{0} / L^{2}$. This laminar solution becomes unstable to transverse large-scale perturbations (on scales much larger than $L$ ) when the Reynolds number $\operatorname{Re} \equiv U L / v$ exceeds the threshold $\operatorname{Re}_{c}=$ $\sqrt{2}$ [15]. While this instability is two dimensional (the Squire theorem), by increasing Re the flow develops further instabilities and eventually becomes three dimensional and turbulent. Here we consider the case $\operatorname{Re} \gg \operatorname{Re}_{c}$ for which linear and weakly nonlinear analyses are not applicable and therefore we will make use of direct numerical simulations (DNSs) of (1).

An interesting property of the Kolmogorov flow is that even in the turbulent regime, the mean velocity has nearly the Kolmogorov profile [2]

$$
\overline{u_{1}(\mathbf{x}, t)}=U \cos (z / L),
$$

where the overbar denotes a space-time average over $x, y$, and $t$. Moreover, the Reynolds stress is also monochromatic

$$
\overline{u_{1} u_{3}}=S \sin (z / L),
$$

with amplitude $S$, and therefore the momentum budget [obtained by averaging (1)] becomes a simple algebraic relation for the coefficients of the monochromatic terms

$$
F=\frac{S}{L}+\frac{\nu U}{L^{2}} .
$$

The friction coefficient $f$ for the Kolmogorov flow can be defined as the ratio between the work done by the force and the kinetic energy of the flow

$$
f=F L / U^{2},
$$

which, because the energy input is simply $\epsilon=\left\langle u_{i} f_{i}\right\rangle=\frac{1}{2} F U$ (angular brackets represent the average over the whole space), is equivalent also to the dissipation factor

$$
f=\frac{2 \epsilon L}{U^{3}} .
$$

We observe in the literature that the dissipation factor is sometimes defined in terms of the root mean square (rms) velocity $U_{\mathrm{rms}}=\left\langle|\mathbf{u}|^{2}\right\rangle^{1 / 2}$ as $\beta=\epsilon L / U_{\mathrm{rms}}$ [16]. Numerical simulations shows that $U_{\mathrm{rms}}$ is proportional to $U$ (see below) and therefore so are $f$ and $\beta$, but an explicit relation between the two dimensionless coefficient is not known. Together with the friction factor, we define also the dimensionless stress coefficient $\sigma \equiv S / U^{2}$ and therefore we can rewrite the momentum budget (4) as

$$
f=\sigma+\frac{1}{\mathrm{Re}} .
$$

In the laminar fix point, for which $S=0$, we have from (7) the usual laminar expression for the friction factor

$$
f_{\text {lam }}=\frac{1}{\mathrm{Re}} .
$$

As Re increases, the laminar solution becomes unstable and the friction factor becomes larger than $f_{\text {lam }}$ and eventually approaches a constant as $\operatorname{Re} \gg 1$. This corresponds to the so-called zeroth law of turbulence [17].

From a mathematical point of view, although $f$ cannot be computed analytically in a turbulent flow, several bounds have been obtained. The simplest lower bound is given by the laminar expression (7), which corresponds to the absence of turbulence. In the case of Kolmogorov flow with periodic boundary conditions, an upper bound for the dissipation factor in the limit of high Reynolds numbers is [18] $\beta \leqslant \beta_{b}=$ $\pi / \sqrt{216} \simeq 0.214$.

\section{RESULTS FROM NUMERICAL SIMULATIONS}

We integrated (1) on a cubic periodic box of size $L_{\mathrm{box}}=2 \pi$ with Kolmogorov forcing at scale $L=1$, fixed viscosity, and different values of forcing amplitude $F$. Starting from a zerovelocity configuration, a turbulent, statistically stationary state is reached after several large-scale eddy turnover times $T$. The value of $F$ determines the amplitude of the velocity in the flow and therefore the Reynolds number as shown in Table I. As $\mathrm{Re} \gg \operatorname{Re}_{c}$ the flow is always in the turbulent regime.

After the flow has reached a stationary condition, we compute the mean profiles from which we obtain $U$ and $S$ by fitting with (2) and (3) and we also measure the other statistical properties of the flow. We remark that the use of a forcing at the smaller wave number generates strong fluctuations in the large-scale properties of the flow, therefore we have to average over many $T$ (between 10 and 100) to have a good convergence of mean quantities. We check the convergence to a statistical steady state by using (7), which is indeed satisfied with good accuracy.

A first remarkable result obtained from our simulations concerns the intensities of the turbulent fluctuations at different Reynolds numbers. We decompose the flow into the mean velocity and fluctuations as $u_{i}(\mathbf{x}, t)=\bar{u}_{i}(z)+u_{i}^{\prime}(\mathbf{x}, t)$ [where $\overline{u_{1}}$ is given by (2) and $\overline{u_{2}}=\overline{u_{3}}=0$ ]. Figure 1 shows that the rms turbulent fluctuations $u_{\text {rms }}^{\prime}$ grows linearly with Re, which is proportional to the mean velocity amplitude $U$. In particular we obtain $u_{\mathrm{rms}}^{\prime} / U \simeq 0.54 \pm 0.03$ in the range of Re investigated. The same behavior is observed for the rms velocity $U_{\text {rms }}$. We find $U_{\text {rms }} / U \simeq 1.10 \pm 0.02$. This result confirms that the friction factors $f$ and $\beta$, which are defined on the basis of $U$ and $U_{\text {rms }}$, respectively, are proportional to each other, as we anticipated in the previous section. Of course we expect different ratios $u_{\text {rms }}^{\prime} / U$ and $U_{\text {rms }} / U$ for much smaller values of Re, close to the instability threshold.

\section{A. Momentum budget}

In Fig. 2 we show the friction coefficient $f=F L / U^{2}$ and the stress coefficient $\sigma=S / U^{2}$ as a function of Re as obtained from the numerical simulations. We find that, for $\operatorname{Re} \gtrsim 160$, 
TABLE I. Parameters of the simulations: $F$ is the amplitude of the forcing, $U$ is the amplitude of the mean profile, $\operatorname{Re}=U L / v, u_{\mathrm{rms}}^{\prime}$ is the rms of the fluctuation of the $x$ component of the velocity, $\epsilon=v\left\langle(\partial \mathbf{u})^{2}\right\rangle$ is the mean energy dissipation, $\eta=\left(v^{3} / \epsilon\right)^{1 / 4}$ is the Kolmogorov scale, $\tau_{\eta}=(v / \epsilon)^{1 / 2}$ is the Kolmogorov time scale, and $T=\left\langle\mathbf{u}^{2}\right\rangle /(2 \epsilon)$ is the large-scale time. The integral scale $L=1$ and the viscosity $v=10^{-3}$ are fixed for all simulation. Simulations up to $\operatorname{Re}=480$ are done at resolution $N=128, \operatorname{Re}=730$ and 990 at $N=256$, and $\operatorname{Re}=1350$ and above at $N=512$. For all the simulations $k_{\max } \eta \geqslant 1$.

\begin{tabular}{lllllrr}
\hline \hline $\operatorname{Re}$ & $F$ & $U$ & $u_{\mathrm{rms}}^{\prime}$ & $\epsilon$ & $\eta$ & $\tau_{\eta}$ \\
\hline 60 & 0.0005 & 0.060 & 0.032 & $1.49 \times 10^{-5}$ & $9.06 \times 10^{-2}$ & 8.21 \\
78 & 0.001 & 0.078 & 0.042 & $3.93 \times 10^{-5}$ & $7.10 \times 10^{-2}$ & 5.05 \\
120 & 0.002 & 0.12 & 0.062 & $1.17 \times 10^{-4}$ & $5.40 \times 10^{-2}$ & 2.92 \\
160 & 0.004 & 0.16 & 0.087 & $3.16 \times 10^{-4}$ & $4.22 \times 10^{-2}$ & 1.78 \\
230 & 0.008 & 0.23 & 0.12 & $9.31 \times 10^{-4}$ & $3.22 \times 10^{-2}$ & 63.1 \\
340 & 0.016 & 0.34 & 0.17 & $2.70 \times 10^{-3}$ & $2.47 \times 10^{-2}$ & 50.2 \\
480 & 0.032 & 0.48 & 0.25 & $7.73 \times 10^{-3}$ & $1.90 \times 10^{-2}$ & 0.61 \\
730 & 0.064 & 0.73 & 0.38 & $2.30 \times 10^{-2}$ & $1.44 \times 10^{-2}$ & 0.36 \\
990 & 0.128 & 0.99 & 0.53 & $6.41 \times 10^{-2}$ & $1.12 \times 10^{-2}$ & 0.21 \\
1350 & 0.256 & 1.35 & 0.76 & $1.73 \times 10^{-1}$ & $8.72 \times 10^{-3}$ & 0.13 \\
2000 & 0.512 & 2.00 & 1.08 & $5.23 \times 10^{-1}$ & $6.61 \times 10^{-3}$ & 0.076 \\
\hline \hline
\end{tabular}

the friction coefficient follows to a good approximation

$$
f=f_{0}+\frac{b}{\operatorname{Re}}
$$

and therefore from (7)

$$
\sigma=f_{0}+\frac{b-1}{\operatorname{Re}}
$$

The fit for $f$ with (9) gives $f_{0}=0.124$. It is interesting to note that in the Kolmogorov flow the asymptotic behavior (9) and (10), which describes the large-Re limit, is already present for relatively small Re. In our set of simulations it can be observed for $\operatorname{Re} \gtrsim 160$, which corresponds to the onset of the fully developed turbulence regime, as we will show in Sec. III D. This is at variance with the case of pipe flows, in which the asymptotic behavior of the drag coefficient appears at much larger Re and the laminar regime is still present for Re in the range investigated in our study. We recall that in pipe flows the laminar regime is linearly stable, while the Kolmogorov flow becomes linearly unstable already at

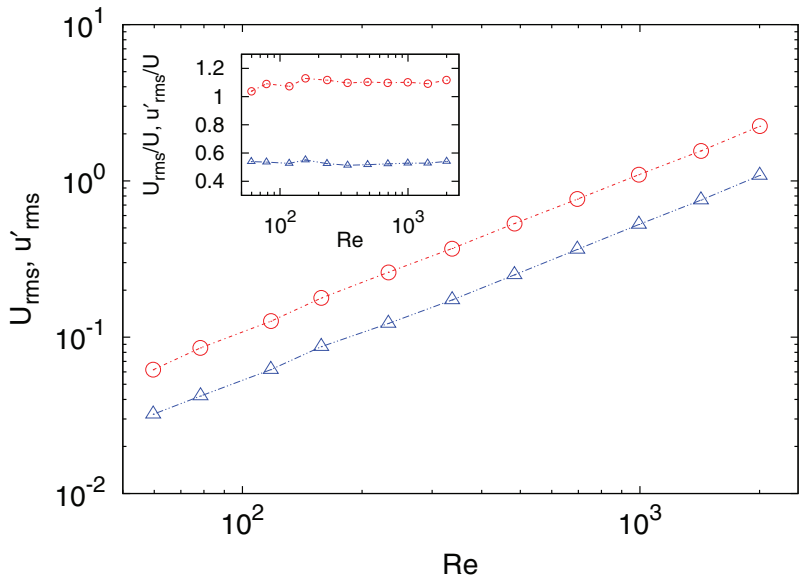

FIG. 1. (Color online) The rms velocity $U_{\text {rms }}$ (red circles) and turbulent fluctuations velocity $u_{\mathrm{rms}}^{\prime}$ (blue triangles) as a function of the Reynolds number $\operatorname{Re}=U L / v$. The inset shows the two rms velocities normalized by the mean velocity amplitude $U$.
$\operatorname{Re}>\sqrt{2}$. The early manifestation of the large-Re asymptotic regime in the Kolmogorov flow is important from the point of view of application because it justifies the extrapolation of large-Re behavior from relatively low-Re simulations.

The dissipation factor $\beta$ is shown in the inset of Fig. 2 as a function of the Reynolds number, here defined in terms of $U_{\text {rms }}$ for consistency with previous literature. While a weak dependence on Re is still observable, numerical data suggest an asymptotic value, as $\operatorname{Re} \rightarrow \infty, \beta \lesssim 0.05$, consistent with but quite smaller than the bound $\beta_{b} \simeq 0.214$.

\section{B. Local energy balance}

In the stationary condition we can write, by multiplying (1) by $u_{i}$ and averaging over $(x, y)$, the energy balance profile

$$
\epsilon_{I}(z) \equiv \overline{u_{i} f_{i}}=\epsilon_{v}(z)+T(z)
$$

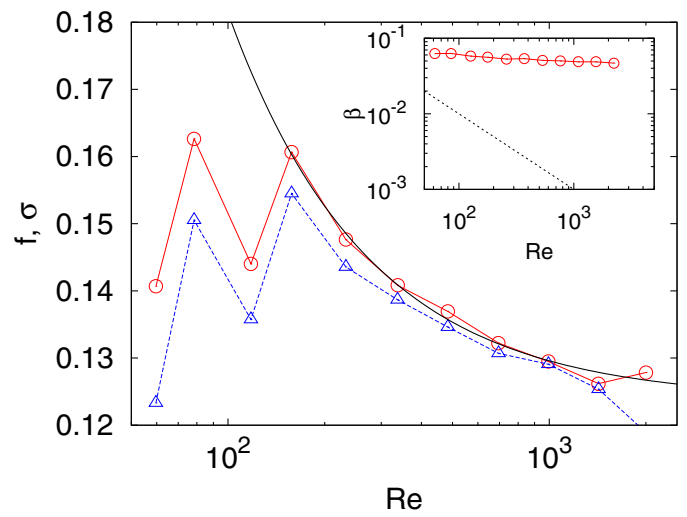

FIG. 2. (Color online) Evolution of the friction coefficient $f=$ $F L / U^{2}$ (red circles) and stress coefficient $\sigma=S / U^{2}$ (blue triangles) as a function of the Reynolds number $\operatorname{Re}=U L / v$. The black line represents the fit with (9), which gives $f_{0}=0.124$ and $b=5.75$. The inset shows the dissipation factor $\beta=\epsilon L / U_{\text {rms }}$ (red circles) vs $\operatorname{Re}=U_{\text {rms }} L / v$ for the set of simulations. The dashed line represents the laminar lower bound $\beta_{\text {lam }}=1 /$ Re. 


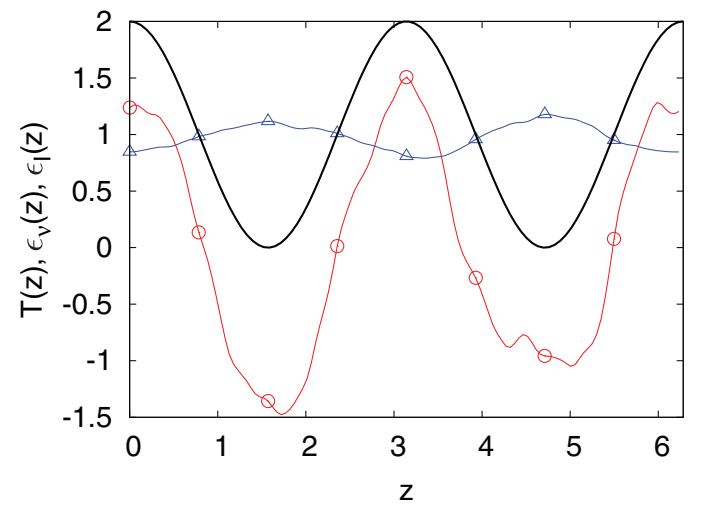

FIG. 3. (Color online) Profiles of energy dissipation $\epsilon_{v}(z)$ (blue line with triangles) and energy transport $T(z)$ (red line with circles), which sum to energy input $\epsilon_{I}(z)$ (black line) according to (11). All quantities have been normalized to the mean energy input $\epsilon_{I}=F U / 2$. The data are from the simulation at $\operatorname{Re}=160$.

where the energy dissipation profile is

$$
\epsilon_{v}(z) \equiv v \overline{|\nabla \mathbf{u}|^{2}}
$$

and $T(z)$ is

$$
T(z)=\partial_{z} \overline{u_{3}\left(u^{2} / 2+p\right)}-v \partial_{z}^{2} \overline{u^{2} / 2} .
$$

Given the monochromatic mean profile for the velocity field, we have

$$
\epsilon_{I}(z)=\frac{F U}{2}[1+\cos (2 z / L)]
$$

with average $\epsilon_{I}=F U / 2=F^{3 / 2}(L / 4 f)^{1 / 2}$.

In (11) $T(z)=\partial_{z} J(z)$ represents the spatial energy transport, which can be $T(z)>0$ where the energy is locally injected and $T(z)<0$ where it is removed. Of course $\langle T(z)\rangle_{z}=0$ for energy conservation (and $\epsilon_{I}=\epsilon_{\nu}$ ).

Figure 3 shows the different terms in (11). Because the dissipation term is almost homogeneous, the transfer term mainly reflects the profile of the energy input (14). A small modulation is observable in the dissipation, which is found to be almost independent of the Reynolds number [2]. By means of a Reynolds decomposition of the velocity field in the mean profile and fluctuating components $u_{i}(\mathbf{x}, t)=\overline{u_{i}(\mathbf{x}, t)}+u_{i}^{\prime}(\mathbf{x}, t)$ (where $\overline{u_{i}} \neq 0$ for $i=1$ only), the energy dissipation (12) can be rewritten as

$$
\epsilon_{v}(z)=v \frac{U^{2}}{L^{2}} \sin ^{2}(z / L)+v \overline{\left(\partial_{j} u_{i}^{\prime}\right)^{2}},
$$

where we have used (2). The first term in (15) represents the direct dissipation by viscosity on the large-scale mean flow, which, when normalized with the mean energy input $F U / 2$, decays as $1 / \mathrm{Re}$. The second term in (15) represents the local dissipation of velocity fluctuations. Its contribution in the energy balance, proportional to velocity gradient, is weakly dependent on $\mathrm{Re}$ and still inhomogeneous in $z$, as shown in Fig. 4. The term is responsible for the modulation observed in Fig. 3, which persists also for larger Reynolds numbers. If we neglect these small modulations and assume as a zeroth-order approximation an homogeneous dissipation $\epsilon_{v}(z)=\epsilon_{v}=F U / 2$, from (11) we obtain an explicit

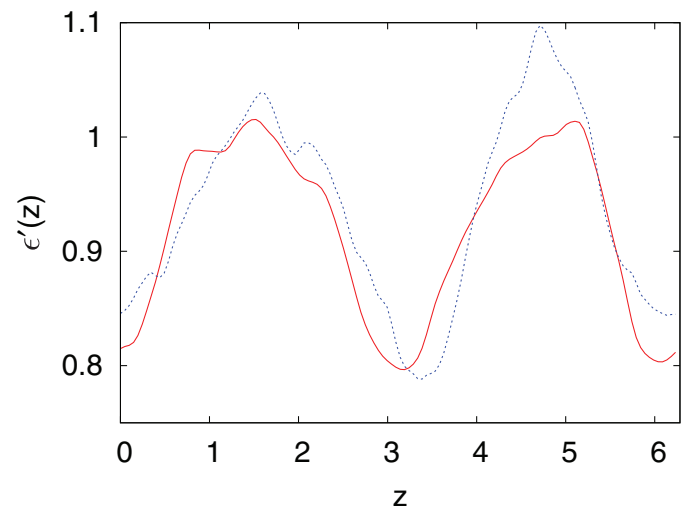

FIG. 4. (Color online) Profiles of local dissipation of the fluctuation energy $\epsilon^{\prime}(z)=v \overline{\left(\partial_{j} u_{i}^{\prime}\right)^{2}}$ normalized with the mean energy input $F U / 2$ for $\operatorname{Re}=78$ (red solid line) and $\operatorname{Re}=160$ (blue dotted line).

expression for the energy transport term

$$
T(z)=\frac{F U}{2} \cos (2 z / L)
$$

\section{Spatial and scale dependence of the energy flux}

We have seen in the previous section that the energy dissipation profile $\epsilon_{v}(z)$ (at small scales) is much more homogeneous than the energy input profile $\epsilon_{I}(z)$ (at large scales). This means that the energy flux that, on average, transfers energy from large to small scales also redistributes energy in space. It is therefore interesting to investigate how the energy is transferred at a different position $z$ at the different scales in the turbulent cascade.

In order to get more insight in this mechanism of energy transfer, we consider the scale-by-scale budget of kinetic energy $[19,20]$. We introduce a filter kernel $G_{\ell}(\mathbf{x})=\ell^{-3} G(\mathbf{x} / \ell)$ [with $\int d^{3} x G(\mathbf{x})=1$ ], which defines a low-pass filtered field by the convolution $u_{i}^{(\ell)}(\mathbf{x}) \equiv\left(G_{\ell} \star u_{i}\right)(\mathbf{x})$. By applying the filter to the equation of motion (1), contracted with $u_{i}$, we get the equation for the energy at large scale

$$
\partial_{t} E^{(\ell)}(\mathbf{x})+\partial_{j} J_{j}^{(\ell)}(\mathbf{x})=-\Pi^{(\ell)}(\mathbf{x})-D^{(\ell)}(\mathbf{x})+F^{(\ell)}(\mathbf{x}),
$$

where $E^{(\ell)}=(1 / 2)\left|\mathbf{u}^{(\ell)}\right|^{2}$ is the large-scale kinetic energy density, $D^{(\ell)}=\nu\left|\nabla \mathbf{u}^{(\ell)}\right|^{2}$ is large-scale energy dissipation, $J_{i}^{(\ell)}=u_{j}^{(\ell)}\left[\tau_{i j}^{(\ell)}+\delta_{i j}\left(E^{(\ell)}+p^{(\ell)}\right)\right]-v \partial_{i} E^{(\ell)}$ is the spatial energy transport at large scales, and

$$
\Pi^{(\ell)}(\mathbf{x})=-\tau_{i j}^{(\ell)} \partial_{j} u_{i}^{(\ell)}
$$

is the scale-to-scale energy flux, where $\tau_{i j}^{(\ell)}=\left(u_{i} u_{j}\right)^{(\ell)}-$ $u_{i}^{(\ell)} u_{j}^{(\ell)}$ is the stress tensor (of the filtered field). The term $\Pi_{\ell}(\mathbf{x})$ represents the local energy flux to scales smaller than $\ell$ at point $\mathbf{x}$.

In our setup we are interested in the horizontally averaged version of (17), which, in stationary conditions and for $\ell$ smaller than the forcing scale $(\ell<L)$, reads

$$
\partial_{z} \overline{J_{3}^{(\ell)}}(z)=-\overline{\Pi^{(\ell)}}(z)-\overline{D^{(\ell)}}(z)+\epsilon_{I}(z) .
$$

When averaged over $z$, the first term in (19) vanishes and one obtain the homogeneous balance for the energy 


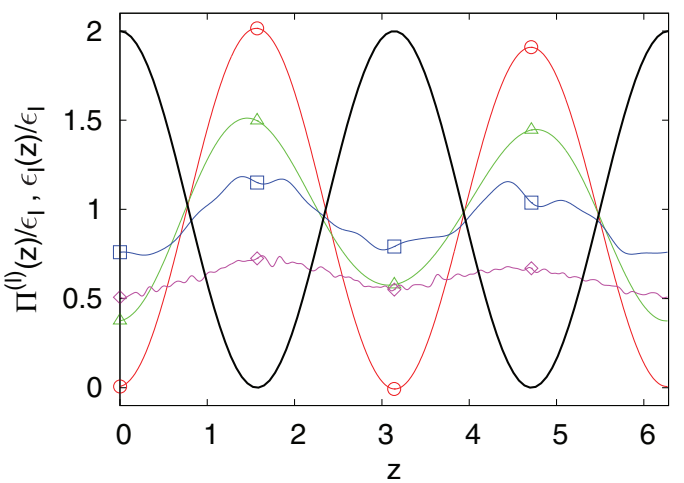

FIG. 5. (Color online) Profile of the scale-to-scale energy flux $\overline{\Pi^{(\ell)}}(z)$ for different scales of the filter $\ell=L / 2$ (red circles), $\ell=$ $L / 4$ (green triangles), $\ell=L / 16$ (blue squares), and $\ell=L / 64$ (pink diamonds) for the run at $\mathrm{Re}=2000$. The black line represents the theoretical energy input $\epsilon_{I}(z)=F U \cos ^{2}(z / L)$. All quantities are normalized with the mean energy input $F U / 2$.

flux

$$
\left\langle\Pi^{(\ell)}\right\rangle=-\left\langle D^{(\ell)}\right\rangle+\epsilon_{I} .
$$

In the inertial range of scales $(L \gg \ell \gg \eta)$ the dissipative term is negligible and one has $\left\langle\Pi^{(\ell)}\right\rangle=\epsilon_{I}$. This mean flux is reduced at smaller scales by the presence of dissipation. Figure 5 shows the energy transport profile $\overline{\Pi^{(\ell)}}(z)$ for different values of the filter scale $\ell$, together with the energy input $\epsilon_{I}(z)$. At the largest scale $(\ell=L / 2)$ the flux is strongly inhomogeneous, while moving to smaller scales it becomes more uniform. In the inertial range of scales the $z$-averaged value of $\overline{\Pi^{(\ell)}}(z)$ is constant (and equal to the input), as shown by the first three curves in Fig. 5. Moving to smaller scales, closer to the dissipative range of scales, the term $D^{(\ell)}(z)$ in (19) is not negligible any more and consequently the average flux decreases.

We find that the profile of the scale-to-scale flux is never negative and vanishes in correspondence with the maximum input at the largest scale. This means that there is no backscatter of energy at a given $z$ in the Kolmogorov channel [while, of course, the one-point flux (18) can be negative]. This remarkable result (which is found to be independent of the Reynolds number) suggests that at the largest scale the $z$-averaged energy transport can be simply expressed as $\overline{\Pi^{(\ell)}}(z) \simeq 2 \epsilon_{I}-\epsilon_{I}(z)$. Using (14) and (19) one ends up with a simple prediction for the profile of the spatial transport at the largest scale

$$
\overline{J_{3}^{(L)}}(z)=\frac{F U L}{2} \sin (2 z / L)
$$

This result has a clear interpretation: $\overline{J_{3}^{(\ell)}}(z)$ represents the current of energy in the $z$ direction. As it redistributes energy among different regions in the channel, it is positive (at larger $z)$ in regions where the input decreases $\left[\partial_{z} \epsilon_{I}(z)<0\right]$ and negative (at smaller $z$ ) in regions where the input increases $\left[\partial_{z} \epsilon_{I}(z)>0\right]$.

\section{Structure functions}

In the inertial range of scales $\eta \ll \ell \ll L$, the amplitude of turbulent velocity fluctuations is expected to exhibit a scaling

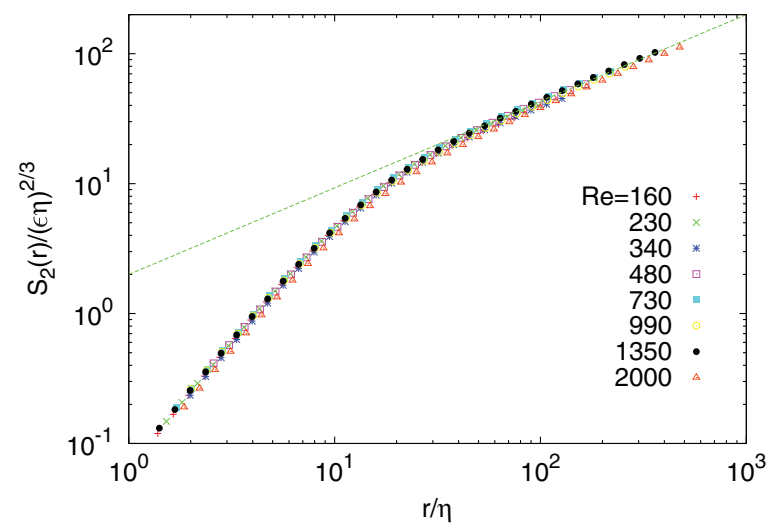

FIG. 6. (Color online) Isotropic second-order structure function $S_{2}(\ell)$ for various $\mathrm{Re}$. The dashed line is the dimensional scaling $S_{2}(\ell) \sim(\epsilon \ell)^{2 / 3}$.

behavior. The scaling behavior of the structure functions $S_{p}(\ell)=\left\langle\left(\delta u_{\ell}\right)^{p}\right\rangle \sim \ell^{\zeta_{p}}$ encodes relevant information on the statistics of longitudinal velocity increments $\delta u_{\ell}=[\boldsymbol{u}(\boldsymbol{x}+$ $\ell)-\boldsymbol{u}(\boldsymbol{x})] \cdot \hat{\ell}$.

In the case of the Kolmogorov flow the structure functions are expected to show a dependence on the coordinate $z$ and the direction $\hat{\ell}$ due to the inhomogeneity and anisotropy of the forcing. In order to extract their homogeneous and isotropic projection we have averaged the structure functions over several isotropically distributed directions $\hat{\ell}$ and over all the available values of the $z$ coordinate.

In Fig. 6 we show the second-order structure function $S_{2}(\ell)$ obtained from our simulations at various Re. We observe good agreement with the dimensional scaling $S_{2}(\ell) \sim(\epsilon \ell)^{2 / 3}$ and a remarkable collapse of the curves when the scales $\ell$ are normalized with the Kolmogorov scale $\eta$ and the amplitude of $S_{2}(\ell)$ is rescaled with the dimensional factor $(\epsilon \eta)^{2 / 3}$. The limited scaling range does not allow us to investigate the presence of intermittency corrections.

The negative sign of the third-order structure functions $S_{3}(\ell)$ in the inertial range signals the direction of the mean energy transfer from large to small scales. The scaling behavior (shown in Fig. 7) is in good agreement with the Kolmogorov

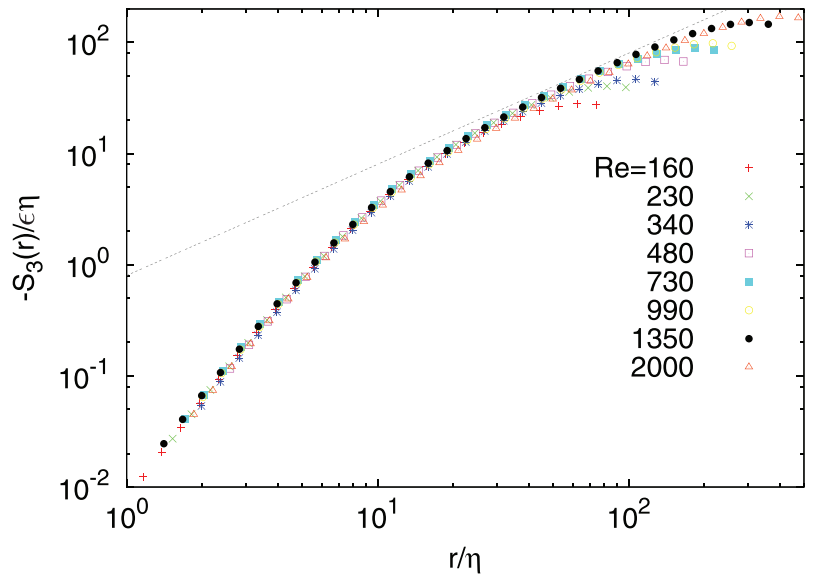

FIG. 7. (Color online) Isotropic third-order structure function $S_{3}(\ell)$ for various $\operatorname{Re}$. The dashed line represents the $\frac{4}{5}$ th law $S_{3}(\ell)=$ $-\frac{4}{5} \epsilon \ell$. 


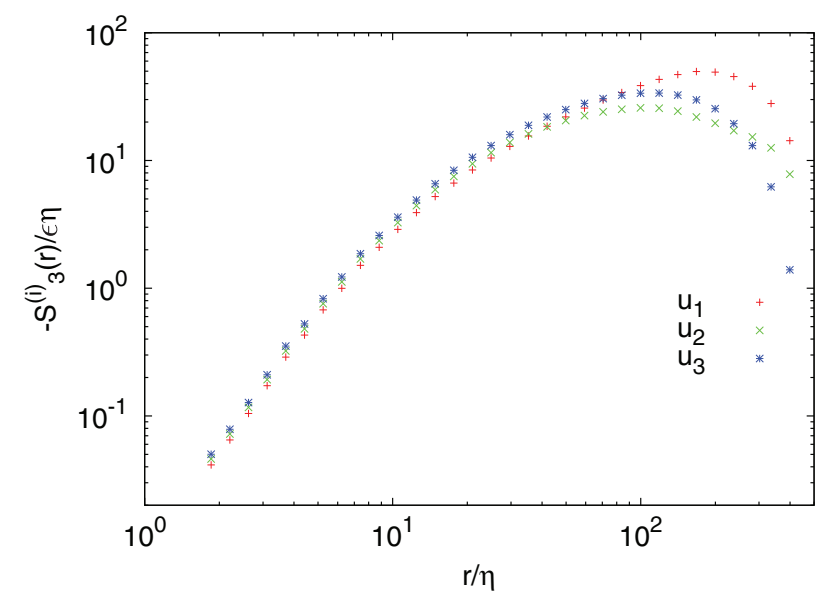

FIG. 8. (Color online) Longitudinal third-order structure function $S_{3}^{(i)}(\ell)$ in the direction $i=1,2,3$ for the run at $\operatorname{Re}=2000$.

$\frac{4}{5}$ th law $S_{3}(\ell)=-\left(\frac{4}{5}\right) \epsilon \ell$. It is interesting to note that $S_{3}(\ell)$ begins to exhibit a scaling range at $\mathrm{Re} \simeq 160$, which is the lowest $\operatorname{Re}$ at which the friction factor $f$ begins to display the asymptotic behavior $f \simeq f_{0}+b / \operatorname{Re}$ (see Fig. 2). Indeed, the zeroth law of turbulence, i.e., the fact that the friction coefficient becomes almost constant as $\operatorname{Re} \rightarrow \infty$, is strictly connected to the development of the turbulent energy cascade.

In Fig. 8 we show the longitudinal third-order structure functions $S_{3}^{(i)}(\ell)$ computed along the directions of the axes $i=1,2,3$. The behavior in the different directions reflects the anisotropy of the flow. In particular the $S_{3}$ in the forced $(i=1)$ direction displays a broader scaling range with respect to the other two, which is consistent with the fact that the flow in this direction is more energetic.

\section{CONCLUSION}

In this paper we have presented an analysis of the momentum end energy balance of the turbulent Kolmogorov flow. By means of a set of direct numerical simulations at increasing Reynolds number we have shown that the rms value of turbulent fluctuations $u_{\text {rms }}^{\prime}$ grows linearly with the amplitude of the mean flow $U$ and that the friction coefficient $f=$ $F L / U^{2}$ follows the asymptotic behavior $f=f_{0}+O\left(\mathrm{Re}^{-1}\right)$ as $\operatorname{Re} \rightarrow \infty$.
We have shown that the local flux of kinetic energy has a strong dependence both on the scale $\ell$ and on the vertical coordinate $z$. The maximum energy flux toward small dissipative scales occurs at the nodes of the Kolmogorov flow, i.e., the regions located on the vertical positions where the mean flow is vanishing and the mean shear is maximum. Conversely, the minimum energy flux is observed at the antinodes where the mean flow is maximum. The amplitude of this spatial modulation of the energy flux reduces as the turbulent cascades proceed toward small scales, but it is still present at dissipative scales.

We have also derived a prediction for the spatial transport of kinetic energy, which describes how kinetic energy is redistributed among different regions of the flow while being transported toward small dissipative scales. In particular we have shown that there is an energy current from the antinodes to the nodes that transports kinetic energy from the regions where the energy input provided by the forcing is maximum, i.e., the maxima of the mean flow, toward the regions where the input vanishes, i.e., the maxima of the mean shear. As a consequence, this current produces a partial recovery of the homogeneity of the flow.

From a theoretical point of view, the Kolmogorov flow represents an ideal framework to investigate the properties of spatial transfer of kinetic energy in nonhomogeneous, sheared turbulent flows. In spite of the absence of material boundaries, it allows us to define mean profiles for all the relevant quantities, e.g., the mean velocity, the mean shear, and the turbulent fluxes. Due to this remarkable feature, it can be used to investigate the interplay between the mean flow and the bulk turbulence, avoiding at the same time the complexities induced by the development of turbulent boundary layers. It provides therefore an ideal tool to study the properties of internal shears in turbulent flows, which appears, e.g., in geophysical currents and jets. In view of possible geophysical applications, it would be very interesting to investigate Lagrangian properties of the Kolmogorov flow, such as the absolute and relative dispersion of tracers.

\section{ACKNOWLEDGMENT}

Computer time provided by Cineca is gratefully acknowledged.
[1] G. I. Sivashinsky, Physica D 17, 243 (1985).

[2] V. Borue and S. Orszag, J. Fluid Mech. 306, 293 (1996).

[3] Z. She, Phys. Lett. A 124, 161 (1987).

[4] S. Fortova, Comput. Math. Math. Phys. 53, 311 (2013).

[5] J. Shebalin and S. Woodruff, Phys. Fluids 9, 164 (1997).

[6] L. Biferale and F. Toschi, Phys. Rev. Lett. 86, 4831 (2001).

[7] B. Legras, B. Villone, and U. Frisch, Phys. Rev. Lett. 82, 4440 (1999).

[8] N. Balmforth and Y. Young, J. Fluid Mech. 450, 131 (2002).

[9] G. Boffetta, A. Celani, A. Mazzino, A. Puliafito, and M. Vergassola, J. Fluid Mech. 523, 161 (2005).

[10] A. Bistagnino, G. Boffetta, A. Celani, A. Mazzino, A. Puliafito, and M. Vergassola, J. Fluid Mech. 590, 61 (2007).

[11] G. Krstulovic, G. Thorner, J.-P. Vest, S. Fauve, and M. Brachet, Phys. Rev. E 84, 066318 (2011).
[12] M. E. Brachet, M. D. Bustamante, G. Krstulovic, P. D. Mininni, A. Pouquet, and D. Rosenberg, Phys. Rev. E 87, 013110 (2013).

[13] G. Boffetta, A. Celani, and A. Mazzino, Phys. Rev. E 71, 036307 (2005).

[14] S. Childress, R. Kerswell, and A. Gilbert, Physica D 158, 105 (2001).

[15] L. D. Meshalkin and Ia. G. Sinai, J. Appl. Math. Mech. 25, 1700 (1961).

[16] C. R. Doering, B. Eckhardt, and J. Schumacher, J. Fluid Mech. 494, 275 (2003).

[17] U. Frisch, Turbulence: The Legacy of A. N. Kolmogorov (Cambridge University Press, Cambridge, 1995).

[18] B. Rollin, Y. Dubief, and C. Doering, J. Fluid Mech. 670, 204 (2011).

[19] M. Germano, J. Fluid Mech. 238, 325 (1992).

[20] G. Eyink, J. Stat. Phys. 78, 335 (1995). 